\title{
An Anxiety Phenomenon in Reading Scientific Articles in Indonesian and English: A Neurolinguistics Analysis
}

\author{
Jatmika Nurhadi* Rosita Rahma, Lestari Kusuma Dewi, Aswan
}

\author{
Universitas Pendidikan Indonesia \\ *Corresponding author. Email: jatmikanurhadi@upi.edu
}

\begin{abstract}
This study aims to describe the activation of anxiety in reading scientific articles in Indonesian and English. Activation of anxiety was assessed based on brain tomography when reading was performed. This study used a oneshot case study. The elements described were the interpretations of the results of the EEG recording, including brain waves and brain maps in the form of brain tomography maps through neurolinguistic analysis using Standardized Low-Resolution Electromagnetic Brain Tomography (sLORETA). This study revealed that the condition of anxiety in reading activities occurs due to cognitive branching, failure to recognize words and failure to encode them into memory. The sLORETA method on reading activities can show the correct localization according to the activated brain function.
\end{abstract}

Keywords: Anxiety, EEG, reading, scientific article, sLORETA

\section{INTRODUCTION}

Reading anxiety is related to several emotional problems that occur while reading. This can be described as a process of the low reading, reluctance, or difficulty reading, which is usually accompanied by repeated 'neutral' experiences, such as reading aloud, ambient distractions, and other causes that make readers associate themselves with negative emotions (Jalongo \& Hirsh, 2010). Reading anxiety is also experienced when reading in a foreign language or when there are unfamiliar/unknown vocabulary, language systems, and material (Saito, Garza \& Horwitz, 1999; Zhao, Guo \& Dynia, 2013). In some cases, reading anxiety is generally classified as situational anxiety. Situational anxiety is caused by new situations, new and unknown information, or changes in events that make a person uncomfortable (Potter et al., 2014).

Biologically, the condition of anxiety is related to amygdala activity. The amygdala is the center for processing fear and anxiety, and its function can be impaired in anxiety disorders. Sensory information enters the amygdala via the basolateral complex nucleus. The basolateral complex processes sensory-related memory and communicate memory and sensory processing elsewhere in the brain, such as the medial prefrontal cortex and sensory cortex. Another vital area is the adjacent central nucleus of the amygdala, which controls emotional responses through connections to the brainstem, hypothalamus, and cerebellum areas. The amygdala area also has more excellent connectivity with the parietal cortex and the prefrontal cortex circuits that underlie executive function (Etkin et al., 2009). In other words, reading anxiety is more active when the brain experiences sensory, and memory overload in the amygdala and other parts of the brain that are connected when reading activity is carried out.

One of the attempts to record the various stimuli and responses that appear in the brain and describe the cognitive and memory structures is by using the electroencephalography (EEG) method. Tatum et al. (2008) stated that the EEG is a unique and meaningful measurement of the electrical function of the brain that graphically displays the difference in voltage from within two brain function locations recorded over time. EEG involves the study of these recordings of electrical signals produced by the brain.

Brodmann's (2006) theory can be used to describe mental representations in the brain. The function of localizing this area will take advantage of several EEG theories. The mental process in acquiring concepts and words in reading activities will be shown through the results of recording electrical signals in the respondent's head, which is called EEG. To describe and interpret the results of recording and EEG measurements on reading activities of people with dyslexia when reading, three theories are used, namely: neurolinguistic theory, brain wave interpretation theory and brain mapping theory. Neurolinguistic theory is adapted from Ahlsen (2006) 
dan Ingram (2007). The brain wave theory was adapted from Stern and Engel (2005) and Tatum et al. (2008), Meanwhile, brain map theory was adapted from Toga and Mazziotta (2002), Craver (2007), and Hirsch and Brenner (2010).

Roberto D. Pascal-Marqui initially proposed the sLORETA method of brain imaging analysis. This method can be formulated as a spatial filter non-adaptive method with a standardized minimum-norm filter output. Standardization makes the sLORETA filter no localization bias (Sekihara \& Nagarajan, 2015, pp. 4647). This method is denoted as standardized lowresolution electromagnetic tomography of the brain. Compared to fMRI and MEG, which have high resolution but have systematic non-zero localization errors, sLORETA has zero localization (zero error). This method is an update from the LORETA Pascual-Marqui, Michel and Lehmann (1994) method that was introduced previously. The sLORETA method was initially carried out by simulation using the Talairach human brain head atlas model, available as a digital MRI form from the Brain Imaging Center, Montreal Neurological Institute (MNI) (Pascual-Marqui, 2002)

\section{METHOD}

This study used a one-shot case study. The interpretation of the results of the EEG recording, including brain waves and brain maps in the form of brain tomography maps through neurolinguistic analysis using Standardized Low-Resolution Electromagnetic Brain Tomography (sLORETA) were described to understand the investigated phenomenon.

\subsection{Participants}

This study involved 20 students (5 male and 15 female) as respondents. All respondents are native speakers of Indonesian and not active speakers of English, from the city of Bandung, Indonesia. All respondents filled out the consent form after receiving an explanation of the series of taking before data collection; the respondents performed a simulation of data collection. The respondents were given two stimuli: (1) reading scientific article texts in Indonesian, and (2) reading scientific article texts in English.

\subsection{Data Collection Procedure}

The data collection technique was carried out by observing the recording and measurement of the EEG through the Open Brain Computer Interface (OPEN BCI) hardware and software. The hardware is a Cyton + Daisy 32 bit Board with 16 channels and $125 \mathrm{~Hz}$ recording samples and is connected via OpenBCI Dongle to the ASUS A442UQ laptop, while the software uses a standalone application from OpenBCI. From these devices, electrical signals in the brain will be recorded through electrodes installed based on the International System 10-20 points, namely: Fp1 (Frontal Polar 1), Fp2,
F3 (Frontal 3), F4, F7, F8, C3 (Central 3), C4, T3 (Temporal 3), T4, T5, T6, P3 (Parietal 3), P4, O1 (Occipital 1), O2, and reference points A1 (Earlobe 1), and $\mathrm{A} 2$.

\subsection{Data Processing Procedures}

The EEG data that were ready were then processed using the LORETA Key v20200709 software. Before EEG data were imported, electrode placement was registered to MNI (Montreal Neurological Institute) coordinates. Then the electrodes were transformed into a matrix (sxyz) for sLORETA analysis. EEG data that had been prepared beforehand were computed into SLORETA (slor). Then the computation results are opened in the LORETA Key Viewer. The EEG.slor data opened in the viewer is then divided into two filters: (1) $15-18 \mathrm{~Hz}$ for attention conditions, and (2) $25-30 \mathrm{~Hz}$ for anxiety conditions. The peak of the EEG.slor data (maximum value) at each frequency is used as the observation point. From the observation point, Brodmann's area and the position of brain localization were analyzed.

\section{FINDINGS AND DISCUSSION}

The condition of anxiety in reading scientific articles in this study will be described through sLORETA-based tomography analysis. The results of the sLORETA analysis are described in the Diagram 1. Based on Figure 1 , it can be described that the most dominant Brodmann area used in anxiety conditions with a frequency filter of $25-30 \mathrm{~Hz}$ in reading scientific articles in Indonesian is Brodmann Area 10 (BA-10) 5 respondents (25\%), Brodmann Area 19 (BA-19) 3 respondents (15\%) and Brodmann Area 37 (BA-37) 3 respondents (15\%), while the English scientific articles are in Brodmann Area 11 (BA-11) and Brodmann Area 18 respectively 3 respondents (15\%). Evidence on the state of anxiety is different from the attention condition, which tends to be concentrated / dominant on activity in BA-18. Meanwhile, in conditions of anxiety, it is more widespread.

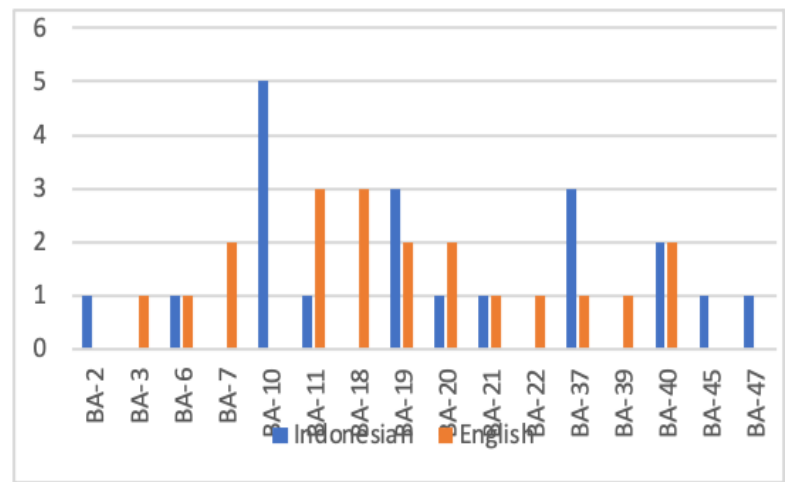

Figure 1 The Position of Brodmann Area Localization on Anxiety Conditions Reading Scientific Articles in Indonesian and English 
The principal activity in Brodmann Area 10 (BA-10) in the anxiety state suggests 'cognitive ramification' processing. Cognitive branching allows previously running tasks to be maintained in a pending state for subsequent retrieval and execution after completing the current task. This condition when reading occurs when the reader tries to do many tasks simultaneously, namely working memory simultaneously, episodic memory, and many tasks in the form of visual activities and encoding to memory. When this section is activated continuously, the activation process leads the reader to a state of anxiety and raises stress levels.

In BA-19, normal activation was associated with visual association, shape recognition, great attention, and multimodal integration. However, excessive activation triggering this part leads to a state of anxiety. For example, when readers repeatedly fail to make attention or fail to recognize visual forms, such as words, this is what causes anxiety to escalate. Similar to BA-19, BA37 has a role in making shape and color recognition. Failure to recognize this process causes mental conditions to increase anxiety. In BA-11, activation is typically performed when the reader encodes new information into long-term memory. However, failure of the encoding process can increase the state of anxiety. Meanwhile, BA-18, under normal conditions, plays a role in conducting visual information flow (visual stream) from the retina to neurons (retinotopic). Receptive failure that occurs in this area makes visual information challenging to encode, which can lead to increased anxiety.

The condition of anxiety is more spread out in different Brodmann Areas. In reading activities in Indonesian, anxiety problems occur because of the branching of cognitive activity. Meanwhile, in English speaking activities, anxiety problems arise due to the failure of vocabulary recognition. This problem happened because the respondents are not native English speakers. The dominant activity in this section is similar to the functions described in previous studies, such as cognitive branching problems (Koechlin \& Hyafil, 2007), problems with memory and word recognition failure (Frey \& Petrides, 2000), and visual encoding problems (Brewer et al., 2005). The difference with previous studies is that in this study, the distribution map is imaged clearly so that the distribution of anxiety to each respondent can be shown based on their reading activities.

\section{CONCLUSION}

This study summarized several findings, namely: (1) the condition of anxiety in reading activities occurs due to cognitive branching, failure to recognize words and failure to encode them into memory, and (2) the sLORETA method on reading activities can show the correct localization according to the activated brain function. The results of these findings can have implications for reading learning. To reduce anxiety to read, the stimulus must reduce overload on cognitive activities, explain new or unfamiliar vocabulary, and train memory skills.

\section{ACKNOWLEDGMENTS}

We would like to thank the LPPM UPI for providing funding for this research.

\section{REFERENCES}

Ahlsén, E. (2006). Introduction to neurolinguistics. Amsterdam: John Benjamins Publishing Company.

Brewer, A. A., Liu, J., Wade, A. R., \& Wandell, B. A. (2005). Visual field maps and stimulus selectivity in human ventral occipital cortex. Nature Neuroscience, $\quad 8(8), \quad 1102-1109$. doi: $10.1038 / \mathrm{nn} 1507$

Brodmann, K. (2006). Brodmann's: Localisation in the cerebral cortex. New York: Springer.

Craver, C. F. (2007). Explaining the brain: Mechanisms and The Mosaic Unity of Neuroscience. Oxford: Oxford University Press.

Etkin, A., Prater, K. E., Schatzberg, A. F., Menon, V., \& Greicius, M. D. (2009). Disrupted amygdalar subregion functional connectivity and evidence of a compensatory network in generalized anxiety disorder. Archives of General Psychiatry, 66(12), 1361-1372.

doi: 10.1001/archgenpsychiatry.2009.104

Frey, S., \& Petrides, M. (2000). Orbitofrontal cortex: A key prefrontal region for encoding information. Proceedings of the National Academy of Sciences of the United States of America, 97(15), 8723 8727. doi: 10.1073/pnas.140543497

Hirsch, L. J., \& Brenner, R. P. (2010). Atlas of EEG in critical care. Oxford: Wiley-Blackwell.

Ingram, J. C. L. (2007). Neurolinguistics: An introduction to spoken language and its disorders. Cambridge: Cambridge University Press.

Jalongo, M. R., \& Hirsh, R. A. (2010). Understanding reading anxiety: New insights from neuroscience. Early Childhood Education Journal, 37(6), 431435. doi: 10.1007/s10643-010-0381-5

Koechlin, E., \& Hyafil, A. (2007). Anterior prefrontal function and the limits of human decision-making. Science, 318(5850), 594-598. doi: 10.1126/science.1142995

Pascual-Marqui, R. D. (2002). Standardized low resolution brain electromagnetic tomography (sLORETA): Technical details. Methods \& Findings in Experimental \& Clinical Pharmacology, 24, 5-12.

Pascual-Marqui, R. D., Michel, C. M., \& Lehmann, D. (1994). Low resolution electromagnetic tomography: A new method for localizing electrical activity in the brain. International Journal of Psychophysiology, 18(1), 49-65. doi: 
10.1016/0167-8760(84)90014-X

Potter, C. M., Wong, J., Heimberg, R. G., Blanco, C., Liu, S. M., Wang, S., \& Schneier, F. R. (2014). Situational panic attacks in social anxiety disorder. Journal of Affective Disorders, 167, 1-7. doi: 10.1016/j.jad.2014.05.044

Saito, Y., Garza, T. J., \& Horwitz, E. K. (1999). Foreign language reading anxiety. Modern Language Journal, 83(2), 202-218. doi: 10.1111/00267902.00016

Sekihara, K., \& Nagarajan, S. S. (2015). Electromagnetic brain imaging. London: Springer.

Stern, J. M., \& Engel, J. (2005). An atlas of EEG patterns. Tucson: Lippincott Williams \& Wilkins.

Tatum, W. O., Husain, A. M., Benbadis, S. R., \&
Kaplan, P. W. (2008). Handbook of EEG interpretation. TX: Demos Medical Publishing.

Toga, A. W., \& Mazziotta, J. C. (2002). Brain mapping: The methods. California: Academic Press.

Zhao, A., Guo, Y., \& Dynia, J. (2013). Foreign language reading anxiety: Chinese as a foreign language in the United States. Modern Language Journal, 97(3), 764-778. doi: 10.1111/j.15404781.2013.12032.x 